\title{
Effects of a short-term detraining period on muscle functionality and cognition of strength-trained older women: a preliminary report
}

\author{
Hélio José Coelho Júnior ${ }^{1 * *}$, Bruno Rodrigues', Ivan de Oliveira Gonçalves², Marco Carlos Uchida' \\ ${ }^{1}$ Faculty of Physical Education, University of Campinas (UNICAMP), Campinas/SP, Brazil \\ ${ }^{2}$ Center of Health Sciences, University of Mogi das Cruzes, Mogi das Cruzes, Brazil
}

The present study aimed to investigate the impact of a 1-month detraining composed by Christmas and New Year's Day in the functional and cognitive parameters of 12 older women who had previously participated in 22-week moderate intensity strength training program $(S T ; n=6)$ or remained performing their regular habits of life during all study period, without engaging in physical exercise programs (CG; $n=6)$. Transfer capacity (i.e., Timed Up and Go [TUG]) and executive function (i.e., TUG with a cognitive task) were increased in the ST group after the interven- tion. However, the 1-month detraining period was enough to totally reverse the gains in both parameters. In conclusion, data of the present study indicate that 1 month of detraining was enough to totally reverse the beneficial effects of a 6-month ST program on physical mobility and executive function of older women.

Keywords: Strength training, Older adults, Exercise training, Resistance training, Cognition, Functionality

\section{INTRODUCTION}

The aging process is a continuous phenomenon accompanied by alterations in some physiological systems, collaborating with the development of geriatric syndromes and chronic diseases. Alterations in the architecture and function of the neuromuscular complex, for example, are leading factor in the etiology of sarcopenia (Papa et al., 2017). This progressive disease is characterized by a marked reduction in muscle mass and strength, as well as functionality (Cruz-Jentoft et al., 2010; Papa et al., 2017), which is strongly associated with a poor prognosis (Coelho Júnior et al., 2015; Kizilarslanoglu et al., 2016; Morley et al., 2011).

Recent data suggested that elevated levels of physical activity have a protective role against sarcopenia (Steffl et al., 2017). Moreover, regarding the clinical signals of sarcopenia, strength training (ST) has been suggested as a powerful tool to combat these parameters in the organic system, reversing and improving muscle atro- phy, dynapenia and loss muscle functionality (Law et al., 2016). In fact, data from original studies (Kalapotharakos et al., 2004; Kalapotharakos et al., 2005; Prestes et al., 2009), confirmed by meta-analytic analyses (Peterson et al., 2010; Peterson et al., 2011; Tschopp et al., 2011), support the aforementioned hypothesis.

On the other hand, contrarious to the gains demonstrated after ST programs, several studies have been investigated whether strength-trained older adults underwent to a period of training cessation, termed as detraining, may present reversal in the beneficial adaptations achieved after exercise training. Most evidence indicate a significant decrease in the gains in physical capabilities and functionality during detraining period in comparison with values obtained after the ST program (Coetsee and Terblanche, 2015; Correa et al., 2013; Correa et al., 2016; Kalapotharakos et al., 2010; Tokmakidis et al., 2009).

However, there is no consensus whether the values are still higher after detraining period in comparison with the pre-exercise
${ }^{*}$ Corresponding author: Hélio José Coelho Júnior

(D) https://orcid.org/0000-0001-7482-9514

Faculty of Physical Education, University of Campinas (UNICAMP), Av. Érico

Veríssimo, 701, Cidade Universitária “Zeferino Vaz", Barão Geraldo - Campinas -

SP, CEP: 13.083-851, Brazil

Tel: +55-11-949398302, Fax: +55-19-35216788, E-mail: coelhojunior@hotmail.com.br

Received: June 9, 2017 / Accepted: September 13, 2017
This is an Open Access article distributed under the terms of the Creative Commons Attribution Non-Commercial License (http://creativecommons.org/licenses/by-nc/4.0/) which permits unrestricted non-commercial use, distribution, and reproduction in any medium, provided the original work is properly cited. 
period, so that data have shown reversion (Coetsee and Terblanche, 2015; Kalapotharakos et al., 2010) and nonreversion (Correa et al., 2013; Correa et al., 2016; Tokmakidis et al., 2009) of the ST-induced adaptations. Moreover, most experiments have discussed if distinctive designs of ST, modulating the variables of exercise training, such as intensity and velocity of muscle contraction (Correa et al., 2016; Henwood and Taaffe, 2008), can differently influence the behavior during detraining.

Nevertheless, little attention has been given to the characteristics of the detraining period. This seems to be important since periods of detraining composed by holidays (Christmas and New Year's Day), may induce a larger number of unhealthy habits with deleterious effects on muscle functionality (Houston et al., 2007; Lorenzo-López et al., 2017; Wirth et al., 2017)—including, but not limited to elevated sedentary behavior and alcohol consumption, than a detraining period without holidays, for example.

Furthermore, it is noteworthy, that there is an increasing number of experiments investigating the impact of exercise training on the cognitive parameters of older adults, including executive function (Coelho Júnior et al., 2017b; Liu-Ambrose et al., 2010). Executive function is an important cognitive domain, which collaborates to the development of essential activities of daily living (ADL), once it allows the individual to create, develop, execute and evaluate the effectiveness of a strategy (Snyder et al., 2015). However, inadequate data exist concerning the influence of a detraining period in these parameters.

Therefore, the present study aimed to investigate the impact of a 1-month detraining composed by Christmas and New Year's Day in the functional and cognitive parameters of strengthtrained older women.

\section{MATERIALS AND METHODS}

\section{Subjects}

Twelve untrained older women (age range, 60-74 years) from two specialized public community health centers for older adults in a town of São Paulo City metropolitan area, in the southeastern Brazil, were recruited in 2015. The inclusion criteria were as follows: female, untrained (Rhea, 2004), and age $\geq 60$ years. Exclusion criteria were: hormone replacement and/or psychotropic drugs use, cardiovascular disease (acute myocardial infarction, stroke, peripheral arterial disease, and transient ischemic disease), pulmonary disease, neurological or psychiatric disease (Parkinson or Alzheimer disease), musculoskeletal disorders, metabolic diseases (diabetes mellitus type II), comorbidities associated with greater risk of falls and recent history of smoking or alcohol abuse. All volunteers signed the informed consent form and completed all measurements. This study was approved by the Research Ethics Committee of the University of Campinas (protocol No. 835.733). This study was developed in accordance with the Declaration of Helsinki and according to Resolution 196/96 of the National Health Council.

\section{Procedures}

The present study has a randomized experimental design, which aimed to investigate the effects of a 1-month detraining in the functional and cognitive parameters of older women who had been previously underwent to a 6-month ST program (Fig. 1).

Therefore, patients were undergoing to functional and cognitive evaluations before and after 22 weeks of ST, as well as after a 1 month of detraining. In summary, after an extensive medical eval-

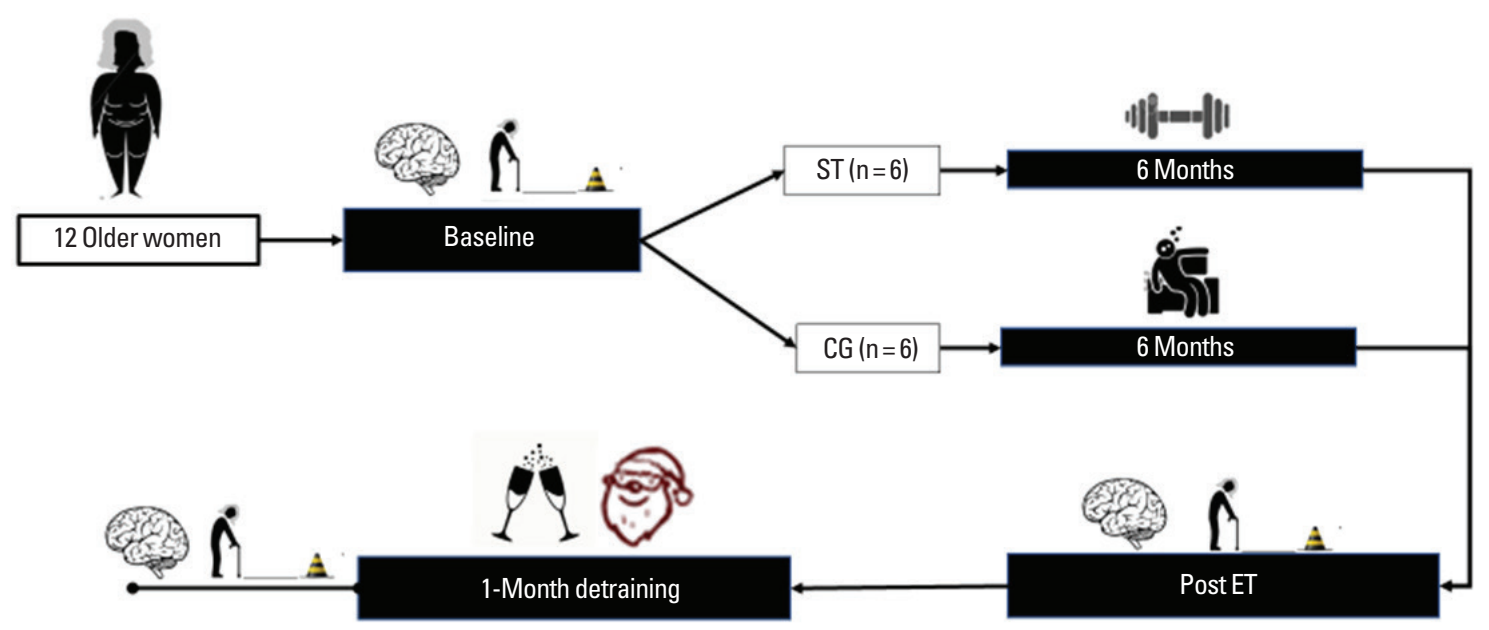

Fig. 1. Experimental design of the presente study. ST, strength training; CG, control group. 
Table 1. Comparison between the groups regarding the morphological and functional parameters

\begin{tabular}{lcc}
\hline Variable & CG & ST \\
\hline Age $(\mathrm{yr})$ & $66.5 \pm 1.5$ & $65.0 \pm 4.1$ \\
Body mass $(\mathrm{kg})$ & $81.2 \pm 11.3$ & $73.6 \pm 7.6$ \\
Body mass index $\left(\mathrm{kg} / \mathrm{m}^{2}\right)$ & $31.4 \pm 2.3$ & $30.1 \pm 3.2$ \\
Waist circumference $(\mathrm{cm})$ & $85.6 \pm 11.9$ & $86.5 \pm 10.8$ \\
Hip circumference $(\mathrm{cm})$ & $106.2 \pm 9.0$ & $98.0 \pm 11.5$ \\
Handgrip strength $(\mathrm{kgf})$ & $20.6 \pm 4.7$ & $25.1 \pm 2.3$ \\
One-leg stand $(\mathrm{sec})$ & $8.5 \pm 12.2$ & $17.0 \pm 3.7$ \\
Usual walking speed (m/sec) & $0.80 \pm 0.11$ & $0.88 \pm 0.17$ \\
Lower limb muscle power $(\mathrm{cm})$ & $9.3 \pm 2.9$ & $9.4 \pm 6.1$ \\
TUG (sec) & $7.9 \pm 2.1$ & $10.1 \pm 1.5$ \\
TUG with a cognitive task $(\mathrm{sec})$ & $10.8 \pm 1.1$ & $11.1 \pm 0.5$ \\
\hline
\end{tabular}

Values are presented as mean \pm standard deviation.

CG, control group; ST, strength training; TUG, Timed Up and Go.

uation - composed by physical and cardiovascular assessmentsolder women were randomized, using a computer-generated list of random numbers, in ST $(n=6)$ and CG $(n=6)$. Subsequently, volunteers were underwent to the specific protocols (i.e., ST or CG) and then a 1-month detraining. Age, anthropometric characteristics, and functional parameters in both groups are shown in Table 1. Briefly, anthropometric characteristics (weight and height) were evaluated using a body weight scale with stadiometer Filizola (Filizola, São Paulo, Brazil) and body circumferences (e.g., waist and hip) were evaluated using a flexible and inextensible anthropometric tape (Sanny, São José dos Campos, Brazil), as described previously (Coelho Júnior et al., 2016).

\section{Exercise protocol}

The exercise protocols were performed from July 2015 to December 2015. During all procedures, including physical training, the temperature in the laboratory was maintained between $21^{\circ} \mathrm{C}$ and $24^{\circ} \mathrm{C}$. Volunteers did not receive diet recommendations; however, they were required not to change their diet or food habits and the ADL during the entire study period. The exercise protocols occurred under the supervision of three experienced researchers, who were responsible for exercise prescription and monitoring of the exercise sessions.

\section{Strength training}

The ST program was performed twice a week, with an interval of $48 \mathrm{hr}$ between training sessions, for 22 weeks. The exercise session of the ST group was based on 3 sets of 8-10 repetitions of each exercise, at the intensity of 5-6, which is considered difficult on the adapted Borg scale (Foster et al., 2001). This intensity rep- resents approximately $70 \%$ of one-repetition maximum (1RM) (Day et al., 2004). One-min rest interval was adopted between each set. Exercise cadence was 2-3 sec and 2 sec for the concentric and eccentric phases, respectively (Garber et al., 2011; Kraemer and Ratamess, 2004). To ensure the cadence of muscle contractions, a researcher was responsible for counting the time and feedback the volunteers during each muscle contraction, indicating if was necessary increase, decrease or maintain the velocity. Exercises were performed by alternating the major groups in the upper and lower extremities (alternating groups). The exercises performed were: (a) squat on the chair $\left(90^{\circ}\right)$, (b) chest press, (c) seated leg curl, (d) frontal raise, (e) calf raise, (f) arm curl, (g) triceps extension, and (h) abdominal crunch. All exercises were performed in the total range of motion. In the beginning of every experimental session, volunteers accomplished a brief warm-up that consisted of one set of 12-15 repetitions of each exercise without weights.

\section{Control group}

The CG remained their regular habits of life during all study period, without engaging in physical exercise programs. To ensure that the volunteers did not engage a physical exercise program, face-to-face or telephonic contact was performed every 15 days.

\section{Detraining period}

During detraining period, volunteers stopped the ST program for one month and returned to their normal daily activities as prior to the ST period (Yasuda et al., 2015). It is noteworthy, that detraining period occurred between December 16th and January 16th, so that evaluations occurred in the first 3 days after vacation cessation (January 17th, 18th, and 19th). This period is characterized by Christmas and New Year's Day holidays. All volunteers reported participating in the festivities.

\section{Functional and cognitive evaluations}

Before the performance of the tests, an experienced researcher detailed the procedures of each test. The volunteers performed all tests twice, and the higher value recorded in each test was used in the analysis. During all tests, verbal encouragement was provided to assure that volunteers reached the best performance possible (except for the TUG with a cognitive test). Evaluations were described previously by our group (Coelho Júnior et al., 2016; Coelho Júnior et al., 2017a, 2017b).

\section{Handgrip strength}

The maximal voluntary contraction was evaluated using a hy- 
draulic hand dynamometer (Jamar, Jackson, MI, USA) while the subjects remained seated in a chair with the shoulders abducted, elbows near the trunk and flexed at $90^{\circ}$, and wrists in a neutral position (thumbs up). The contralateral arm remained relaxed under the thigh. To determine handgrip strength, the volunteers performed a maximal contraction during 4 sec with the dominant hand.

\section{One-leg stand test}

The one-leg stand test was performed with the volunteers standing in a unipodal stance with the dominant lower limb, the contralateral knee remaining flexed at $90^{\circ}$, the arms remaining crossed in front of the chest, and the head was straight. A stopwatch (Moure Jar, Beijing, China) was activated when the volunteer raised their contralateral foot off the floor and was stopped when the contralateral foot touched the floor again.

\section{Sit-to-stand test}

Volunteers were requested to rise from a chair five times as fast as possible with their arms crossed in front of the body. The stopwatch was activated when the volunteer raised their buttocks off the chair and was stopped when the volunteer seated back.

\section{Walking speed test}

To measure walking speed, a 10-m walking speed test was performed. Volunteers were required to walk a distance of $12 \mathrm{~m}$ at their usual and fastest possible cadences (without running). Before the evaluation, both feet of each volunteer were to remain on the starting line. The measurement was initiated when a foot reached the 1-m line and was stopped when a foot reached the 11-m line. The 1-m intervals at the beginning and end were used to avoid early acceleration and/or deceleration.

\section{Countermovement jump}

The countermovement jump was performed to evaluate leg power. In the initial position, the volunteers stood on a jump platform (Jump System Pro, Cefise, Nova Odessa, Brazil), their feet remained approximately parallel at shoulder width, and their hands rested on their hips. When instructed, the volunteers flexed their knees at approximately $90^{\circ}$ and jumped the maximum height possible.

\section{TUG test}

The TUG test involved getting up from a chair without the help of the arms, walking a distance of $3 \mathrm{~m}$ around a marker placed on the floor, coming back to the same position, and sitting back on the chair. The test began when a researcher shouted a "go!" command. The stopwatch was activated when the volunteers got up from the chair and was stopped when they have seated again.

\section{Cognitive evaluation - Executive function TUG cognitive test}

TUG cognitive test (TUGcog) was accomplished to evaluate executive function. This test is performed equally the conventional TUG; however, a cognitive task (verbal fluency, animal category) must be accomplished during the motor task. Therefore, after the signal of the evaluator, the volunteer performed the route- stand up from the chair, walk $3 \mathrm{~m}$, turn around, walk $3 \mathrm{~m}$ back, and sit down again —naming as many animals as he/she could remember. This task was performed it aloud, allowing the evaluators confirmed if the volunteers were, in fact, accomplishing the task. The time expands to perform the task was recorded to evaluation.

\section{Statistical analyses}

Normality of data was tested using the Kormonov-Smirnov test. Comparisons between the groups for subjects' characteristics and functional parameters were performed using two-sided unpaired Student $t$-test. A two-way analysis of variance followed by a Dunnett post hoc test were performed to identify differences among the different times of evaluations (i.e., baseline, postexercise training and after 1 month of detraining) and treatments (i.e., ST and CG). The effect size was classified according to Rhea (2004). The level of significance was $5 \%(P<0.05)$ and all procedures were performed using IBM SPSS Statistics ver. 20.0 (IBM Co., Armonk, NY, USA). The power of the sample size was determined using $G^{*}$ Power version 3.1.9.2. The intention-to-treat principle was applied to the analysis of the outcomes for all participants based on their assigned treatment. All values are shown as mean \pm standard deviation.

\section{RESULTS}

Subjects did not show adverse events during exercise sessions and evaluations, as well as they were not absenting for more than three exercise sessions. Volunteers did not report any changes in food intake during the whole course of the present study. Table 1 shows the baseline comparisons among ST and CG. As expected, volunteers for both groups were older. Body mass index values and anthropometric analyses (i.e., waist circumference and hip 

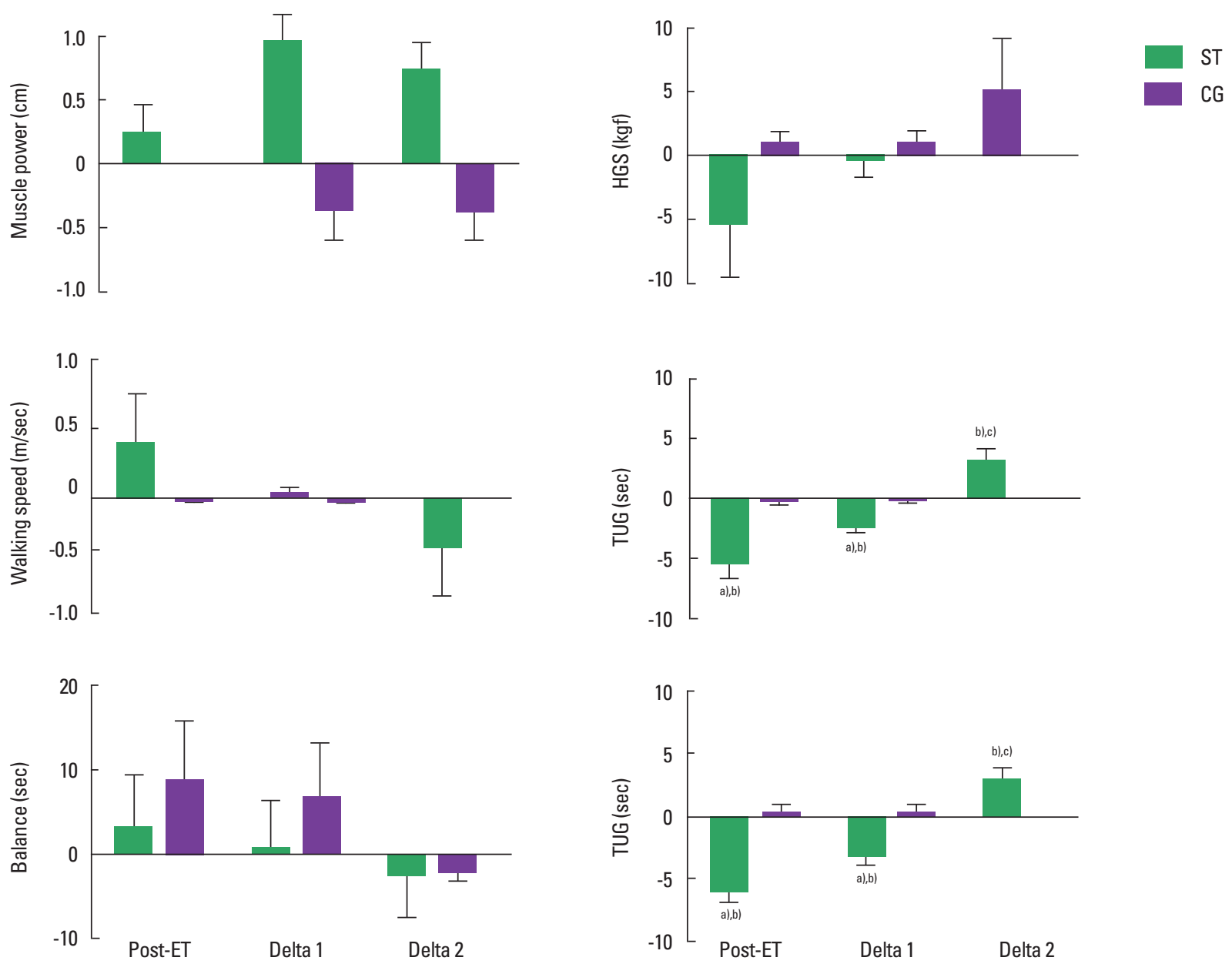

Fig. 2. Delta $(\Delta)$ values of the intragroup and intergroup comparisons among post-ET versus baseline values (i.e., post-ET), 1-month detraining versus baseline values (i.e., delta 1) and 1-month detraining versus post-ET (i.e., delta 2) in ST and CG. ST, strength training; CG, control group; ET, exercise training. ${ }^{\text {al) }} P<0.05$ vs. baseline. ${ }^{b} P<$ 0.05 vs. correspondent time in CG. ${ }^{\mathrm{cl}} P<0.05$ vs. post-ET.

circumference) indicate that most older women were obese. However, there were no significant differences between the groups regarding morphological and functional parameters.

The delta $(\Delta)$ values of the intragroup and intergroup comparisons among post exercise training (ET) versus baseline values (i.e., post-ET), 1-month detraining versus baseline values (i.e., delta 1) and 1-month detraining versus post-ET (i.e., delta 2) in ST and CG are presented in Fig. 2. Handgrip strength, balance, and walking speed were not altered in any evaluation. TUG and TUGcog were improved post-ET and delta 1 in ST, but not in CG. However, a significant decrease was observed in both evaluations after 1-month of detraining in ST. Intergroup comparisons demonstrated that results were lower during post-ET and delta 1 , as well as higher during delta 2 in ST in comparison with CG.

Table 2 shows the ES for functional and cognitive parameters of the ST and CG. ES results are in line with data from hypothesis test, once trivial and small classifications were found in handgrip strength, one-leg stand, usual walking speed and lower limb muscle power tests in both groups at post-ET, delta 1, and delta 2 moments. Moreover, in CG, TUG, and TUGcog were just classified as trivial, whereas a large classification was observed in ST.

\section{DISCUSSION}

Data of the present study indicate that 1 month of detraining was enough to totally reverse the beneficial effects of a 6-month ST program on physical mobility (i.e., TUG) and executive function (i.e., TUGcog) of older women. Therefore, our findings indicate that is necessary be careful with the time without exercise, as generally occur during vacation, once values in delta 2 -1-month 
Table 2. ES for functional and cognitive parameters of the ST and CG

\begin{tabular}{|c|c|c|}
\hline Parameter & CG & ST \\
\hline \multicolumn{3}{|c|}{ Handgrip strength (kgf) } \\
\hline Post-ET & 0.50 (small) & 0.91 (small) \\
\hline Delta 1 & 0 (trivial) & 0.68 (small) \\
\hline Delta 2 & 0.50 (small) & 1.12 (small) \\
\hline \multicolumn{3}{|c|}{ One-leg stand (sec) } \\
\hline Post ET & 0.75 (small) & 0.75 (small) \\
\hline Delta 1 & 0.71 (small) & 0.71 (small) \\
\hline Delta 2 & 0.91 (small) & 0.91 (small) \\
\hline \multicolumn{3}{|c|}{ Usual walking speed (m/sec) } \\
\hline Post-ET & 0 (trivial) & 0.21 (trivial) \\
\hline Delta 1 & 0 (trivial) & 0.18 (trivial) \\
\hline Delta 2 & 0 (trivial) & 0.38 (trivial) \\
\hline \multicolumn{3}{|c|}{ Lower limb muscle power (cm) } \\
\hline Post ET & 0 (trivial) & 0.04 (trivial) \\
\hline Delta 1 & 0.61 (small) & 0.11 (trivial) \\
\hline Delta 2 & 0.61 (small) & 0.34 (trivial) \\
\hline \multicolumn{3}{|l|}{ TUG (sec) } \\
\hline Post-ET & 0 (trivial) & 2.69 (large) \\
\hline Delta 1 & 0 (trivial) & 1.92 (large) \\
\hline Delta 2 & 0.30 (trivial) & 2.88 (large) \\
\hline \multicolumn{3}{|c|}{ TUG with a cognitive task (sec) } \\
\hline Post ET & 0.15 (trivial) & 3.89 (large) \\
\hline Delta 1 & 0 (trivial) & 3.28 (large) \\
\hline Delta 2 & 0 (trivial) & 1.02 (large) \\
\hline
\end{tabular}

Values are presented as mean \pm standard deviation.

CG, control group; ST, strength training; ET, exercise training; TUG, Timed Up and Go; Delta 1, 1-month detraining vs. baseline values; Delta 2, 1-month detraining vs. post-ET.

detraining versus post-ET—-were elevated for TUG and TUGcog.

Regarding physical mobility, data of current study corroborate with several experiments in the literature, which demonstrated decreased muscle functionality after different detraining periods (e.g., 6, 12, and 16 weeks) (Coetsee and Terblanche, 2015; Correa et al., 2013; Correa et al., 2016; Kalapotharakos et al., 2010; Tokmakidis et al., 2009). However, just few studies have demonstrated a total reversal of the adaptations in physical mobility after a detraining period (Coetsee and Terblanche, 2015; Kalapotharakos et al., 2010), so that most studies have indicated that the beneficial effects of ST are only partially reversed after detraining period, and values are still higher after detraining in comparison with the pre-exercise period (Correa et al., 2013; Correa et al., 2016; Tokmakidis et al., 2009).

In fact, as in the present study, Coetsee and Terblanche (2015), for example, demonstrated that the significant improvements observed after the ST program in TUG performance were totally lost during the detraining periods. TUG is a useful test to predict falls risk in older adults, once its performance is composed by, at least, three physical capabilities, to quote: muscle strength, muscle power and balance (Alexandre et al., 2012; Barry et al., 2014). Thus, improvements-and thus also decline-in TUG performance are commonly associated with alterations in one, or more, of these paraments.

Interestingly, our data did not demonstrate significant alterations in muscle power and balance after the detraining period, which could indicate that the decrease in muscle strength was the key factor responsible for this phenomenon. However, lower body muscle strength was not measured. Moreover, it is important to mention that Coetsee and Terblahnche (2015) refuted this hypothesis because the lower TUG performance found after detraining was not followed by reduced lower body muscle strength. The inconsistencies in the findings could be a function of the differences in the initial state of the volunteers, the design of ST program (e.g., frequency, volume, duration, and intensity), as well as the detraining periods (Coetsee and Terblanche, 2015). Accordingly, is not possible indicate the crucial factors that influenced the decline on TUG performance.

Nevertheless, it is noteworthy that most studies have not specified the exactly time where the detraining period occurred. Furthermore, detraining has been quantifying just in the end of a determined period (e.g., 12 months), and interim analyses have not been carried out. This seems to be a valuable information, once short-term detraining periods compose by holidays celebrations (e.g., Christmas and New Year's Day), as was addressed in the current study, may be associated with a larger relative time of sedentary behavior, intake of high calorie foods — composed, predominately, by high fat and low protein-, and alcohol consumption, if compared with long-term detraining periods offered during other periods, for example.

In fact, such behaviors, mainly sedentary behavior and malnutrition, are strongly associated with several important detrimental health outcomes, including increased inflammatory state, muscular atrophy, dynapenia, which interreacts directly and/or indirectly with muscle functionality (Houston et al., 2007; Lorenzo-López et al., 2017; Wirth et al., 2017). Therefore, the alterations observed in the current study after the detraining period may be cause by an inadequate short-term behavior than by differences in ST variables (e.g., exercise intensity and volume). However, we are not able to confirm these inferences and future experiments must investigate the impact of distinct kinds of detraining periods on muscle functionality. 
Regarding executive function, data of the current study demonstrated that the ameliorated performance on TUGcog observed after ST was totally reversed during the detraining period. Executive function is a cognitive capacity composed of other cognitive domains (e.g., shifting, working memory, inhibition), which allow the subject to create, develop and performed a strategy to perform an aim, evaluate the outcomes and, if necessary, change the strategy during or at the end of the task, creating new strategies for the future (Snyder et al., 2015). Consequently, executive function is an essential cognitive domain to the maintenance of autonomy and independence during aging, and its impairment are observed in intermediate conditions (e.g., mild cognitive impairment), as well as during several psychopathological conditions, including Alzheimer disease (Borges et al., 2015).

For the best of our knowledge, this is the first study that investigated the effects of a detraining period on cognitive parameters of older adults. Indeed, most studies have been evaluating the impact of exercise training on executive function of older adults (Coelho Júnior et al., 2017b; Liu-Ambrose et al., 2010) and data about the detraining period are limited, which strongly limits our discussion.

Cognitive resilience refers to the protective capacity of the central nervous system to coping with stressful conditions, such as pathophysiological conditions, and socioeconomic and psychosocial difficulties avoiding that the stressor agent leads the organic system to work at a level below that it was working before (Fontes and Neri, 2015). In the current study, detraining period may be indicated as a stressful condition, once, during this period, the organic system must cope with several elements that may cause a reduction in the increased cognitive capacity acquired after exercise training.

Several factors have been associated with cognitive resilience, including psychological and social factors (e.g., good quality relationships), education level, spiritual and religious beliefs, genetic variants (e.g., CREB1 and APOE4 genotypes) and all these have potential to collaborate in the phenomenon observed during detraining (Fontes and Neri, 2015; Wolf et al., 2017). However, once our executive function assessment was dependent of a high muscular component, is possible infer that alterations on muscular functionality during detraining may exert a key role in the decreasing of executive function. Moreover, we cannot rule out the possibility that the same elements that contribute with muscular declining during detraining have acted in executive function.

In conclusion, data of the present study indicate that 1 month of detraining was enough to totally reverse the beneficial effects of a 6-month ST program on physical mobility (i.e., TUG) and executive function (i.e., TUGcog) of older women.

\section{CONFLICT OF INTEREST}

No potential conflict of interest relevant to this article was reported.

\section{REFERENCES}

Alexandre TS, Meira DM, Rico NC, Mizuta SK. Accuracy of Timed Up and Go Test for screening risk of falls among community-dwelling elderly. Rev Bras Fisioter 2012;16:381-388.

Barry E, Galvin R, Keogh C, Horgan F, Fahey T. Is the Timed Up and Go test a useful predictor of risk of falls in community dwelling older adults: a systematic review and meta-analysis. BMC Geriatr 2014;14:14.

Borges Sde M, Radanovic M, Forlenza OV. Functional mobility in a divided attention task in older adults with cognitive impairment. J Mot Behav 2015;47:378-385.

Coelho Júnior HJ, Aguiar SDS, Gonçalves IdO, Sampaio RAC, Uchida MC, Moraes MR, Asano RY. Sarcopenia is associated with high pulse pressure in older women. J Aging Res 2015;(2015):Article ID 109824.

Coelho Júnior HJ, Rodrigues B, Aguiar SD, Gonçalves IO, Pires FO, Asano RY, Uchida MC. Hypertension and functional capacities in community-dwelling older women: a cross-sectional study. Blood Press 2017a;26:156-165.

Coelho Júnior HJ, Rodrigues B, Feriani DJ, Gonçalves IO, Asano RY, Aguiar SD, Uchida MC. Effects of multicomponent exercise on functional and cognitive parameters of hypertensive patients: a quasi-experimental study. J Aging Res 2017b;2017:1978670.

Coelho Júnior HJ, Sampaio RA, Gonçalvez IO, Aguiar SD, Palmeira R, Oliveira JF, Asano RY, Sampaio PY, Uchida MC. Cutoffs and cardiovascular risk factors associated with neck circumference among community-dwelling elderly adults: a cross-sectional study. Sao Paulo Med J 2016;134:519-527.

Coetsee C, Terblanche E. The time course of changes induced by resistance training and detraining on muscular and physical function in older adults. Eur Rev Aging Phys Act 2015;12:7.

Correa CS, Baroni BM, Radaelli R, Lanferdini FJ, Cunha Gdos S, Reischak-Oliveira Á, Vaz MA, Pinto RS. Effects of strength training and detraining on knee extensor strength, muscle volume and muscle quality in elderly women. Age (Dordr) 2013;35:1899-1904.

Correa CS, Cunha G, Marques N, Oliveira-Reischak Ã, Pinto R. Effects of strength training, detraining and retraining in muscle strength, hypertrophy and functional tasks in older female adults. Clin Physiol Funct 
Imaging 2016;36:306-310.

Cruz-Jentoft AJ, Baeyens JP, Bauer JM, Boirie Y, Cederholm T, Landi F, Martin FC, Michel JP, Rolland Y, Schneider SM, Topinková E, Vandewoude M, Zamboni M; European Working Group on Sarcopenia in Older People. Sarcopenia: European consensus on definition and diagnosis: Report of the European Working Group on Sarcopenia in Older People. Age Ageing 2010;39:412-423.

Day ML, McGuigan MR, Brice G, Foster C. Monitoring exercise intensity during resistance training using the session RPE scale. J Strength Cond Res 2004;18:353-358.

Fontes AP, Neri AL. Resilience in aging: literature review. Cien Saude Colet 2015;20:1475-1495.

Foster C, Florhaug JA, Franklin J, Gottschall L, Hrovatin LA, Parker S, Doleshal P, Dodge C. A new approach to monitoring exercise training. J Strength Cond Res 2001;15:109-115.

Garber CE, Blissmer B, Deschenes MR, Franklin BA, Lamonte MJ, Lee IM, Nieman DC, Swain DP; American College of Sports Medicine. American College of Sports Medicine position stand. Quantity and quality of exercise for developing and maintaining cardiorespiratory, musculoskeletal, and neuromotor fitness in apparently healthy adults: guidance for prescribing exercise. Med Sci Sports Exerc 2011;43:13341359.

Henwood TR, Taaffe DR. Detraining and retraining in older adults following long-term muscle power or muscle strength specific training. J Gerontol A Biol Sci Med Sci 2008;63:751-758.

Houston DK, Cesari M, Ferrucci L, Cherubini A, Maggio D, Bartali B, Johnson MA, Schwartz GG, Kritchevsky SB. Association between vitamin D status and physical performance: the InCHIANTI study. J Gerontol A Biol Sci Med Sci 2007;62:440-446.

Kalapotharakos VI, Diamantopoulos K, Tokmakidis SP. Effects of resistance training and detraining on muscle strength and functional performance of older adults aged 80 to 88 years. Aging Clin Exp Res 2010;22:134-140.

Kalapotharakos VI, Michalopoulou M, Godolias G, Tokmakidis SP, Malliou PV, Gourgoulis V. The effects of high- and moderate-resistance training on muscle function in the elderly. J Aging Phys Act 2004;12: 131-143.

Kalapotharakos VI, Michalopoulos M, Tokmakidis SP, Godolias G, Gourgoulis V. Effects of a heavy and a moderate resistance training on functional performance in older adults. J Strength Cond Res 2005;19: 652-657.

Kizilarslanoglu MC, Kilic MK, Dogrul RT, Sumer F, Varan HD, Kuyumcu ME, Yesil Y, Halil M. Sarcopenia and length of hospital stay. Is this a vicious cycle? Eur J Clin Nutr 2016;70:863.

Kraemer WJ, Ratamess NA. Fundamentals of resistance training: pro- gression and exercise prescription. Med Sci Sports Exerc 2004;36:674688.

Law TD, Clark LA, Clark BC. Resistance exercise to prevent and manage sarcopenia and dynapenia. Annu Rev Gerontol Geriatr 2016;36:205228.

Liu-Ambrose T, Nagamatsu LS, Graf P, Beattie BL, Ashe MC, Handy TC. Resistance training and executive functions: a 12-month randomized controlled trial. Arch Intern Med 2010;170:170-178.

Lorenzo-López L, Maseda A, de Labra C, Regueiro-Folgueira L, Rodríguez-Villamil JL, Millán-Calenti JC. Nutritional determinants of frailty in older adults: a systematic review. BMC Geriatr 2017;17:108.

Morley JE, Abbatecola AM, Argiles JM, Baracos V, Bauer J, Bhasin S, Cederholm T, Coats AJ, Cummings SR, Evans WJ, Fearon K, Ferrucci L, Fielding RA, Guralnik JM, Harris TB, Inui A, Kalantar-Zadeh K, Kirwan BA, Mantovani G, Muscaritoli M, Newman AB, Rossi-Fanelli F, Rosano GM, Roubenoff R, Schambelan M, Sokol GH, Storer TW, Vellas B, von Haehling S, Yeh SS, Anker SD; Society on Sarcopenia, Cachexia and Wasting Disorders Trialist Workshop. Sarcopenia with limited mobility: an international consensus. J Am Med Dir Assoc 2011;12:403-409.

Papa EV, Dong X, Hassan M. Skeletal Muscle Function Deficits in the Elderly: Current Perspectives on Resistance Training. J Nat Sci 2017;3. pii: e272.

Peterson MD, Rhea MR, Sen A, Gordon PM. Resistance exercise for muscular strength in older adults: a meta-analysis. Ageing Res Rev 2010;9:226-237.

Peterson MD, Sen A, Gordon PM. Influence of resistance exercise on lean body mass in aging adults: a meta-analysis. Med Sci Sports Exerc 2011;43:249-258.

Prestes J, Shiguemoto G, Botero JP, Frollini A, Dias R, Leite R, Pereira G, Magosso R, Baldissera V, Cavaglieri C, Perez S. Effects of resistance training on resistin, leptin, cytokines, and muscle force in elderly post-menopausal women. J Sports Sci 2009;27:1607-1615.

Rhea MR. Determining the magnitude of treatment effects in strength training research through the use of the effect size. J Strength Cond Res 2004;18:918-920.

Snyder HR, Miyake A, Hankin BL. Advancing understanding of executive function impairments and psychopathology: bridging the gap between clinical and cognitive approaches. Front Psychol 2015;6:328.

Steffl M, Bohannon RW, Sontakova L, Tufano JJ, Shiells K, Holmerova I. Relationship between sarcopenia and physical activity in older people: a systematic review and meta-analysis. Clin Interv Aging 2017;12: 835-845.

Tokmakidis SP, Kalapotharakos VI, Smilios I, Parlavantzas A. Effects of detraining on muscle strength and mass after high or moderate inten- 
sity of resistance training in older adults. Clin Physiol Funct Imaging 2009;29:316-319.

Tschopp M, Sattelmayer MK, Hilfiker R. Is power training or conventional resistance training better for function in elderly persons? A meta-analysis. Age Ageing 2011;40:549-556.

Wirth K, Klenk J, Brefka S, Dallmeier D, Faehling K, Roqué I Figuls M, Tully MA, Giné-Garriga M, Caserotti P, Salvà A, Rothenbacher D, Denkinger M, Stubbs B; SITLESS consortium. Biomarkers associated with sedentary behaviour in older adults: a systematic review. Ageing
Res Rev 2017;35:87-111.

Wolf C, An Y, Tanaka T, Bilgel M, Gonzalez C, Kitner Triolo M, Resnick S. Cross-sectional and longitudinal effects of CREB1 genotypes on individual differences in memory and executive function: findings from the BLSA. Front Aging Neurosci 2017;9:142.

Yasuda T, Fukumura K, Iida H, Nakajima T. Effects of detraining after blood flow-restricted low-load elastic band training on muscle size and arterial stiffness in older women. Springerplus 2015;4:348. 